\title{
Shape-dependence of the thermal and photochemical reactions of methanol on nanocrystalline anatase $\mathrm{TiO}_{2}$
}

\author{
David A. Bennett ${ }^{1}$, Matteo Cargnello ${ }^{2}$, Benjamin T. Diroll ${ }^{3 \#}$, \\ Christopher B. Murray ${ }^{3}$, and John M. Vohs ${ }^{1}$ \\ ${ }^{1}$ Department of Chemical and Biomolecular Engineering and ${ }^{3}$ Department of Chemistry, \\ University of Pennsylvania, Philadelphia, Pennsylvania 19104 \\ ${ }^{2}$ Department of Chemical Engineering, Stanford University, Stanford, California 94305 \\ ${ }^{\#}$ Present address: Center for Nanoscale Materials, Argonne National Laboratory, Lemont, Illinois 60439
}

\begin{abstract}
Structure-activity relationships and the influence of particle size and shape on the partial- and photo-oxidation of methanol on nanocrystalline anatase $\mathrm{TiO}_{2}$ was investigated using temperature-programmed desorption. The study employed two distinct nanoparticle morphologies: truncated bipyramids exposing primarily $\{101\}$ facets, and flatter platelets exposing primarily $\{001\}$ surfaces, whose nominal sizes ranged from 10 to $25 \mathrm{~nm}$. The platelets were found to be more active for thermally-driven reactions, such as coupling of methoxide groups to produce dimethyl ether, and deoxygenation to produce methane. A dependence of the reactivity of $\{001\}$ facets for the coupling of methoxide groups to produce dimethyl ether on facet size was also observed. In contrast to the thermally-driven reactions, the bipyramidal nanoparticles were observed to be more active for a range of photochemical reactions, including oxidation and coupling to produce methyl formate, and photo-decomposition of surface methoxide species. This study also shows how well-defined nanocrystals can be used to help bridge the materials gap between studies of single crystal model catalysts and their high surface area industrial analogs.
\end{abstract}

keywords: anatase, $\mathrm{TiO}_{2}, \mathrm{TPD}$, methanol, photo-oxidation 


\section{Introduction}

Surface science studies of well-defined, single-crystal, model catalysts have made many important contributions to our understanding of the reactivity of a range of catalytic materials. Indeed, this approach has been invaluable in elucidating structure-activity relationships, and the identification of specific active sites, reaction pathways, and the structure of adsorbed intermediates [1-4]. While this approach has been very successful, it still has its limitations since single crystals often do not contain the range of sites that occur in the analogous high surface area catalysts that are used in industrial practice. Recently studies have started to appear in the literature in which an effort has been made to bridge this so called "materials gap" [5-12]. One promising approach to achieving this goal is use thin films of well-defined nanocrystals as model catalysts. This approach has the advantage that the exposed crystal planes are still well-defined which can allow comparisons to be made to the single crystal literature, if available, while at the same time providing for a wider range of sites, such as those at edges and corners, whose concentration can be systematically controlled by varying the crystallite size and shape. Using well-defined nanocrystals may in many cases also allow one to systematically study the reactivity of individual exposed crystal planes for materials for which single crystals are not readily available, which is the case for many metal oxides.

A recent study by the Overbury group at Oak Ridge National Laboratory of the catalytic activity of ceria nanocrystals (octahedra, cubes, and nanowires) provides a good example of this type of study [5]. In this work it was found that ceria nanowires had higher activity than the octahedral nanocrystals for the conversion of acetaldehyde to both decomposition and coupling products in a flow reactor. Furthermore, the wires and cubes were more selective for C-C bond scission to produce $\mathrm{C}_{1}$ products, while octahedra were more selective for aldol condensation. 
This was attributed, in part to the cerium cations with lower coordination numbers and a higher defect density on the surface of the nanowires and cubes.

We have also recently used this approach to study structure-activity relationships for both catalytic and photocatalytic reactions on anatase $\mathrm{TiO}_{2}\left(\mathrm{~A}-\mathrm{TiO}_{2}\right)$ using methanol as a prototypical oxygenate reactant [13]. This study made use of size-selected, $\mathrm{A}-\mathrm{TiO}_{2}$ nanocrystals with a truncated bipyramidal shape that were cast into thin films on oxidized silicon wafers. As shown in Figure 1, these $\mathrm{A}-\mathrm{TiO}_{2}$ crystals expose the $\{101\}$ family of crystal planes on the sides of the pyramids and are capped by $\{001\}$ planes. Temperature-programmed desorption (TPD) studies of the thermal and photo-oxidation of methanol were used to probe how the crystallite size affected reactivity. As will be discussed in more detail below, we observed that in the absence of UV illumination the nanocrystals were active for the coupling of adsorbed methoxide groups to produce dimethyl ether which occurred primarily on undercoordinated Ti cation sites located on the $\{001\}$ planes. UV illumination caused methyl formate to also be produced which appeared to occur on sites located on the $\{101\}$ planes. The relative yield of this photochemical product was also found to be dependent on the crystallite size.

In the work reported here we have extended our previous study to include the reactions and photoreactions of methanol over $\mathrm{TiO}_{2}$ nanocrystals that have the platelet geometry shown in Figure 1. Note that this shape is just a more truncated version of the bipyramids that were used is our previous study, but due to the different aspect ratio the area of the $\{001\}$ planes relative to the $\{101\}$ planes is much higher in the platelets compared to the bipyramids.

\section{Materials and Methods}


A detailed description of the synthesis of the $\mathrm{TiO}_{2}$ nanocrystals can be found in Gordon et al [14]. Briefly, a stock solution of $\mathrm{TiF}_{4}$ and $\mathrm{TiCl}_{4}$ was added to a cosurfactant (either oleylamine or 1-octadecanol) and heated to $563 \mathrm{~K}$. After seed crystal formation, additional stock solution was delivered via syringe pump. Nanocrystal size was controlled by varying the amount of added stock solution. The nanocrystal shape was determined by the relative surface energies of the $\{101\}$ and $\{001\}$ facets. The $\{001\}$ surface is stabilized in the presence of HF generated during hydrolysis of the $\mathrm{TiF}_{4}$ precursor. Thus oleylamine, which moderates the amount of HF, favors the growth of the $\{101\}$ surfaces and leads to a bipyramidal shape, while 1-octadecanol promotes a higher fraction of exposed $\{001\}$ surfaces, leading to a flatter, platelet shape (see Figure 1). In the current study three different sizes of platelet nanocrystals were used which had lateral dimensions of $14 \mathrm{~nm}, 18 \mathrm{~nm}$, and $22 \mathrm{~nm}$ as determined by electron microscopy. (These values have an accuracy of $\pm 3 \mathrm{~nm}$ ). We also compare to data obtained previously for bipyramidal nanocrystals which had long axes of $10 \mathrm{~nm}, 18 \mathrm{~nm}$, and $25 \mathrm{~nm}$ [13]. To allow the use of traditional surface science analytical techniques, the nanocrystals were suspended in hexane and then spin-cast onto oxidized, single crystal Si substrates to produce thin films.

Transmission electron microscopy (TEM) was used to characterize the as-synthesized nanocrystals. For these experiments the nanocrystals were dispersed onto a 300 mesh C-coated $\mathrm{Cu}$ TEM grid and images were collected using a JEOL JEM1400 TEM operating at $120 \mathrm{kV}$. The thin film samples were also characterized by scanning electron microscopy (SEM) using a JEOL 7500F HRSEM. For these experiments the samples were not coated but a conducting tape was applied near the edge of the substrate to reduce sample charging. Imagines were collected in secondary electron imaging mode with a $5 \mathrm{kV}$ beam. 
Reactivity experiments were conducted in an ultra-high vacuum (UHV) chamber with a base pressure of $2 \times 10^{-10}$ Torr. The chamber was equipped with a quadrupole mass spectrometer (Stanford Research Systems) which was used for temperature programmed desorption (TPD) experiments, and a dual-anode X-ray source (VG Microtech) and a hemispherical electron energy analyzer (Leybold-Heraeus) which were used for x-ray photoelectron spectroscopy (XPS). The thin film samples were cut into pieces approximately $8 \mathrm{~mm} \times 6 \mathrm{~mm}$ and mounted in Ta foil holders which were spot welded to an electrical feedthrough on the UHV chamber. Each sample was heated resistively by passing electrical current through the Ta foil and cooled by conduction from a liquid nitrogen reservoir in contact with the electrical feedthrough. The sample temperature was monitored by a type-K thermocouple which was fixed to the back of the $\mathrm{Si}$ substrate using ceramic adhesive (Aremco).

HPLC-grade methanol (Fisher Scientific) was used as the reactant and was subjected to multiple freeze-pump-thaw cycles before use. The methanol was stored in a glass vial and was admitted to the chamber through a variable leak valve (Varian) and directed onto the sample by a dosing needle positioned near the surface of the sample. The sample was held at $160 \mathrm{~K}$ during methanol dosing. The sample temperature was ramped upwards at $3 \mathrm{~K} \mathrm{~s}^{-1}$ during TPD experiments. Oxygen (Matheson, 99.997\%) was used for sample cleaning and was admitted to the chamber in the same fashion as the methanol.

To study photochemical reactions the samples were illuminated with monochromatic UV light $(365 \mathrm{~nm}$ ) from a UV LED source (Prizmatix). This light was admitted into the chamber via a fiber optic cable and the sample was positioned in front of the end of this cable during UV illumination. The photon flux at the surface of the sample was $10^{15} \mathrm{~cm}^{-2} \mathrm{~s}^{-1}$ as determined by a 
photodiode detector (Thorlabs). The samples were held at $160 \mathrm{~K}$ during illumination to prevent desorption of surface species.

In order to allow quantitative comparison of the product yields from different thin film samples, desorption peak areas were scaled to account for mass spectrometer sensitivity factors and for exposed surface area. The latter was estimated using methanol TPD data as a function of methanol coverage to determine the point at which condensed methanol multilayers first form. The following masses were monitored during TPD experiments: $16\left(\mathrm{CH}_{4}\right), 18\left(\mathrm{H}_{2} \mathrm{O}\right), 30\left(\mathrm{CH}_{2} \mathrm{O}\right)$, $31\left(\mathrm{CH}_{3} \mathrm{OH}\right), 45\left(\mathrm{CH}_{3} \mathrm{OCH}_{3}\right)$, and $60\left(\mathrm{HCOOCH}_{3}\right)$.

\section{Results}

\subsection{Catalyst Characterization}

TEM micrographs of one size of each of the platelet and bipyramidal $\mathrm{A}-\mathrm{TiO}_{2}$ nanocrystals are displayed in Figure $2 \mathrm{a}$ and $2 \mathrm{~b}$, respectively. In these images the bipyramidal crystallites are laying on one of the $\{101\}$ planes while the platelets are situated with the $\{001\}$ surfaces parallel to the image plane. SEM images of spin-cast films of each crystallite morphology are displayed in Figures $2 \mathrm{c}$ and $2 \mathrm{~d}$. These images were obtained after the samples were annealed in UHV at $800 \mathrm{~K}$. The crystallites in the films have a random orientation and the image of the platelet film (Figure 2d) shows that the crystallites in this particular sample have a thickness of $\sim 4 \mathrm{~nm}$. These images also show that in both instances the nanocrystals completely cover the substrate to a depth of at least several particles and that heating to $800 \mathrm{~K}$ does not result in any appreciable sintering of the particles.

XPS analysis of freshly-prepared bipyramidal and platelet thin film samples showed that the surfaces of the nanocrystals were covered with both fluorine and carbon. The fluorine results 
from the use of $\mathrm{TiF}_{4}$ as one of the precursors during synthesis. Chlorine was not detected via XPS. It was found, however, that both of the $\mathrm{F}$ and $\mathrm{C}$ impurities could be removed by annealing in $10^{-8}$ Torr of oxygen at $750 \mathrm{~K}$. Ti( $\left.2 \mathrm{p}\right) \mathrm{XP}$ spectra obtained after this treatment were indicative of $\mathrm{Ti}^{+4}$. Representative XP spectra are provided in the Supplemental Information.

\subsection{Thermal Reactions}

Representative TPD results for the platelet nanocrystals are given in Figure 3a. This dataset was obtained from a thin film of the $14 \mathrm{~nm}$ nanocrystals dosed with $2.5 \mathrm{~L}$ (1 Langmuir $=10^{-6}$ Torr $s$ ) of methanol $\left(\mathrm{CH}_{3} \mathrm{OH}\right)$, an exposure that was found to be sufficient to saturate the surface with methanol. The TPD data show that the majority of the methanol desorbs intact in a broad two-peak structure between 180 and $450 \mathrm{~K}$. Two smaller methanol peaks are also present at 500 $\mathrm{K}$ and $620 \mathrm{~K}$. The only reaction products detected were $\mathrm{H}_{2} \mathrm{O}$ which desorbed at $320 \mathrm{~K}$, and formaldehyde $\left(\mathrm{CH}_{2} \mathrm{O}\right)$, methane $\left(\mathrm{CH}_{4}\right)$ and dimethyl ether $\left(\mathrm{CH}_{3} \mathrm{OCH}_{3}\right)$ which were all produced near $640 \mathrm{~K}$. The $\mathrm{H}_{2} \mathrm{O}$ is likely a byproduct of deprotonation of a fraction of the adsorbed methanol to produce adsorbed methoxide groups and surface hydroxyl groups which incorporate a surface lattice oxygen. There are two competing reaction pathways for these hydroxyl groups: (1) recombination with methoxide to produce methanol or (2) reaction to produce $\mathrm{H}_{2} \mathrm{O}$, both of which occur at $320 \mathrm{~K}[15,16]$. The methoxide groups that don't recombine to produce methanol remain on the surface and give rise to the higher-temperature products in the TPD.

The methanol and $\mathrm{CH}_{2} \mathrm{O}$ products near $640 \mathrm{~K}$ can be attributed to a $\mathrm{C}-\mathrm{H}$ bond scission in an adsorbed methoxide to produce formaldehyde with the resulting $\mathrm{H}$ reacting with another methoxide to produce methanol [17]. In addition to this disproportionation reaction, coupling of adsorbed methoxide groups gives rise to the $\mathrm{CH}_{3} \mathrm{OCH}_{3}$ product at $640 \mathrm{~K}$. As will be discussed 
below, for the reaction of methanol on rutile- $\mathrm{TiO}_{2}\left(\mathrm{R}-\mathrm{TiO}_{2}\right)$ this pathway has been attributed to the reaction of two methoxides adsorbed on a common surface Ti cation [18]. Finally, deoxygenation of adsorbed methoxides produces $\mathrm{CH}_{4}$, also near $640 \mathrm{~K}$. TPD data for the $18 \mathrm{~nm}$ and $22 \mathrm{~nm}$ platelets were qualitatively similar to those in Figure $3 \mathrm{a}$ for the $14 \mathrm{~nm}$ platelets and relative product yields for all three crystallite sizes are given in Table 1.

It is useful to compare these results with what we have reported previously for the reaction of methanol on thin films of the bipyramidal nanoparticles [13]. Representative methanol TPD results obtained from $10 \mathrm{~nm}$ bipyramids are given in Figure 3b. The data in both panels of Figure 3 have been scaled to account for differences in surface area, as described in the experimental section. The relative product yields for two sizes of pyramids are also given in Table 1. These data show that while there are some similarities for the two nanocrystal morphologies, there are also some differences. The similarities include the methanol desorption curves which contain broad two-peak structures between 200 and $400 \mathrm{~K}$ along with smaller peaks near 500 and $650 \mathrm{~K}$, $\mathrm{H}_{2} \mathrm{O}$ production at $320 \mathrm{~K}$, and $\mathrm{CH}_{3} \mathrm{OCH}_{3}$ and $\mathrm{CH}_{4}$ production at $640 \mathrm{~K}$. The relative yields of both $\mathrm{CH}_{3} \mathrm{OCH}_{3}$ and $\mathrm{CH}_{4}$ on the bipyramids, however, was significantly less than that on the platelets.

The data in Table 1 show that for the platelets the yield of both $\mathrm{CH}_{4}$ and $\mathrm{CH}_{2} \mathrm{O}$ does not change appreciably with platelet size, after accounting for differences in the surface area of each sample. However, the yield of $\mathrm{CH}_{3} \mathrm{OCH}_{3}$ shows some size dependence. Figure 4 compares the $\mathrm{CH}_{3} \mathrm{OCH}_{3}$ yield during methanol TPD for both the platelet and bipyramidal samples as a function of the crystallite size. Note that for the platelets the $14 \mathrm{~nm}$ crystallites produced markedly more $\mathrm{CH}_{3} \mathrm{OCH}_{3}$ than the larger crystallites. Also, a similar size dependency was not observed for the bipyramids. 


\subsection{Photochemical Reactions}

Methanol TPD experiments were also used to investigate the photocatalytic activity of the $\mathrm{A}-\mathrm{TiO}_{2}$ nanocrystals. As described in the experimental section, for these experiments methanoldosed samples at $160 \mathrm{~K}$ were exposed to $10^{15} \mathrm{~cm}^{-2} \mathrm{~s}^{-1}$ of $365 \mathrm{~nm}$ UV photons for a set time prior to each TPD run. Representative TPD results obtained following a $3 \mathrm{~h}$ UV exposure on both platelet $(14 \mathrm{~nm})$ and bipyramidal $(10 \mathrm{~nm})$ samples dosed with $2.5 \mathrm{~L}$ methanol are displayed in Figures $5 \mathrm{a}$ and $5 \mathrm{~b}$, respectively. This data shows that the most significant change upon UV exposure is the production of the coupling product methyl formate $\left(\mathrm{HCOOCH}_{3}\right)$ which desorbs between 230 and $300 \mathrm{~K}$. The yield of $\mathrm{HCOOCH}_{3}$, however, is roughly the same on the $10 \mathrm{~nm}$ bipyramidal and $14 \mathrm{~nm}$ platelet nanocrystals following a $3 \mathrm{~h}$ illumination period. UV exposure also increases the $\mathrm{CH}_{4}$ yield and attenuates the $\mathrm{CH}_{3} \mathrm{OCH}_{3}$ yield on the $10 \mathrm{~nm}$ bipyramids, but has little effect on the corresponding yields on the platelets.

While the data in Figure 5 indicates that the $\mathrm{HCOOCH}_{3}$ yield after a $3 \mathrm{~h}$ of $\mathrm{UV}$ exposure is roughly equivalent on the $10 \mathrm{~nm}$ bipyramids and on the $14 \mathrm{~nm}$ platelets, different trends in the production of this product as a function of the UV exposure were observed for the two crystallite morphologies. This is illustrated in Figure 6, which plots the $\mathrm{HCOOCH}_{3}$ yield as a function of nanocrystal size versus UV exposure for the two different types of crystallites. For the platelets the $\mathrm{HCOOCH}_{3}$ yield increases linearly with UV exposure. In contrast, for the bipyramids the yield initially increases with UV exposure, but after $2 \mathrm{~h}$ of UV exposure levels off or decreases. We have previously attributed this decrease to photodecomposition of a portion of the $\mathrm{HCOOCH}_{3}$ product [13]. Also note that the platelets, regardless of size, have a $\mathrm{HCOOCH}_{3}$ yield that is similar to the $10 \mathrm{~nm}$ bipyramids, but much less than the $25 \mathrm{~nm}$ bipyramids. 
Analogous yield trends for $\mathrm{CH}_{4}$ are given in Figure 7. As reported previously [13], a small amount of $\mathrm{CH}_{4}$ is produced on the bipyramids in the absence of UV exposure, but the yield increases five- to ten-fold after $3 \mathrm{~h}$ of UV illumination. This suggests the existence of both thermal and photochemical pathways for $\mathrm{CH}_{4}$ production on these nanocrystals. The platelets (Figure 7a), however, exhibit a much smaller increase in $\mathrm{CH}_{4}$ yield with increasing UV exposure, suggesting the photochemical pathway is less significant on the platelets. On the other hand, $\mathrm{CH}_{4}$ yields are still roughly comparable between the two shapes due to the much higher activity for the thermal pathway on the platelets. Finally, photochemically-produced $\mathrm{CH}_{4}$ yield does not change significantly with platelet size.

\section{Discussion}

To facilitate comparisons between the reactivity of the bipyramidal and platelet anatase nanocrystals, let us first review the results we reported previously for the bipyramidal system. Briefly, we found that the thermal and photochemical reactivity of methanol on the bipyramids which preferentially expose the $\{101\}$ family of planes was similar to that reported for reaction on single crystal $\mathrm{A}-\mathrm{TiO}_{2}(101)$ [13]. Methanol adsorbs molecularly on the fivefold coordinate $\mathrm{Ti}$ cations exposed on this surface and desorbs below $400 \mathrm{~K}$ without undergoing any detectible reactions. However, upon exposure to UV light a portion of the adsorbed methanol undergoes photo-oxidation and coupling to produce $\mathrm{HCOOCH}_{3}[13,19]$. As shown in Figure 3b, in addition to these pathways which can be attributed to the $\{101\}$ surfaces, on the bipyramidal nanocrystals a small fraction of the methanol adsorbs dissociatively to produce methoxide groups which convert to $\mathrm{CH}_{4}$ or undergo a coupling reaction near $640 \mathrm{~K}$ to produce $\mathrm{CH}_{3} \mathrm{OCH}_{3}$. The data indicated that these pathways occur primarily on sites located on the $\{001\}$ capping planes. 
While data for the reaction of methanol on single crystal $\mathrm{A}-\mathrm{TiO}_{2}(001)$ surfaces is not available in the literature, the production of $\mathrm{CH}_{4}$ and $\mathrm{CH}_{3} \mathrm{OCH}_{3}$ near $640 \mathrm{~K}$ during methanol TPD experiments has also been observed for $\{114\}$-faceted $\mathrm{R}-\mathrm{TiO}_{2}(001)$ by Kim and Barteau [18]. Furthermore, these researchers demonstrated that the active site for $\mathrm{CH}_{3} \mathrm{OCH}_{3}$ production are exposed fourfold coordinate Ti cations and proposed that multiple coordination vacancies facilitate the coupling reaction by allowing two methoxide groups to bond to a common Ti site. As noted in the introduction, we have previously suggested that the active sites for $\mathrm{CH}_{3} \mathrm{OCH}_{3}$ production on the bipyramidal nanocrystals are located on the $\{001\}$ capping planes. While fourfold coordinated $\mathrm{Ti}^{4+}$ cations are not present on the ideal $\mathrm{A}-\mathrm{TiO}_{2}-(001)$ surface, this surface is unstable and undergoes a $(1 \mathrm{x} 4)$ reconstruction $[20,21]$. The precise structure of this reconstruction is still subject to some debate [21-25], but a model proposed by Lazzeri and Selloni [26], in which $\mathrm{TiO}_{3}$ species periodically replace rows of the bridging oxygens on the $(1 \mathrm{x}$ 1) surface, is the most energetically favorable and the most consistent with the available TPD data [23]. This reconstruction does contain fourfold coordinated $\mathrm{Ti}^{4+}$ but they are situated such that one of the coordination vacancies is not accessible to adsorbates. Thus, one would not expect the $\mathrm{A}-\mathrm{TiO}_{2}(001)-(4 \times 1)$ surface to be active for the coupling of methoxide groups to produce $\mathrm{CH}_{3} \mathrm{OCH}_{3}$. Note, however, that the surface unit cell for this reconstruction spans $1.5 \mathrm{~nm}$ which is approaching the size of the $\{001\}$ caps in the bipyramidal nanocrystals we have studied which vary from $\sim 3-6 \mathrm{~nm}$. We have suggested that the small size of the $\{001\}$ surfaces relative to the length scale of this reconstruction may result in a different equilibrium structure for the $\{001\}$ caps on the bipyramidal nanocrystals than that observed for bulk single-crystal surfaces and that this, yet to be determined, reconstruction exposes fourfold coordinated $\mathrm{Ti}^{4+}$ cations that are active for the coupling of methoxide groups to produce $\mathrm{CH}_{3} \mathrm{OCH}_{3}$ [18]. 
If this hypothesis is correct and the reconstruction of the (001) surface differs between very small facets (as on the caps of the bipyramids) and macroscopic single-crystalline anatase, one might expect the activity of the $\{001\}$ facets in the nanocrystals to be crystallite-size dependent. As shown in Figure 4 this is indeed the case for the platelets where the yield of $\mathrm{CH}_{3} \mathrm{OCH}_{3}$ was found to decrease with increasing crystallite size. At first glance this conclusion may appear to be at odds with the bipyramid data in this figure, which does not show the same trend. Note, however, that these data are normalized to the total surface area of each nanocrystal film. If the yield data is instead normalized to the surface area of the $\{001\}$ facets in each film and plotted versus the area of the (001) facet in each crystallite as is done in Figure 8, a strong dependence of $\mathrm{CH}_{3} \mathrm{OCH}_{3}$ yield during methanol TPD on the $\{001\}$ facet size becomes apparent with the small $\{001\}$ facets of the bipyramidal crystallites being much more active than those of the larger facets in the platelets. While we acknowledge that with only the limited data presented here this conclusion is somewhat speculative, it does provide some support for the idea that reconstruction of oxide surfaces may in some cases depend on facet size. Of course other factors may also be playing a role here such as defect concentrations (e.g. oxygen vacancies) that vary with crystallite size and shape.

In contrast to the trends in $\mathrm{CH}_{3} \mathrm{OCH}_{3}$ production, the yield of thermally-produced $\mathrm{CH}_{4}$ during methanol TPD is not very sensitive to particle size (see the $0 \mathrm{~h}$ points in Figure 7), but still depends on the nanocrystal shape with the platelets exhibiting higher yields than the bipyramids. Since the ratio of $\{001\}$ to $\{101\}$ surface area is roughly constant for a given nanocrystal shape, the most straightforward explanation for this trend is that the thermal pathway for $\mathrm{CH}_{4}$ production occurs on the $\{001\}$ facets which are predominantly exposed on the platelets. A similar conclusion was obtained in our previous study of the reactivity of the bipyramids [13]. 
Also note that Kim and Barteau have proposed that $\mathrm{CH}_{4}$ production on $\mathrm{R}-\mathrm{TiO}_{2}$ proceeds via deoxygenation of adsorbed methoxides on surface oxygen vacancies $[16,18,27]$. The fact that the oxygen vacancy formation energy is $0.3 \mathrm{eV}$ less on the $\mathrm{A}-\mathrm{TiO}_{2}(001)$ compared to the A$\mathrm{TiO}_{2}(101)$ [28] provides further support that the reaction that produces $\mathrm{CH}_{4}$ from methoxide groups on the $\mathrm{A}-\mathrm{TiO}_{2}$ nanocrystals proceeds primarily on sites on the exposed $\{001\}$ surfaces. Finally $\mathrm{CH}_{2} \mathrm{O}$ was produced via dehydrogenation of methoxide groups near $640 \mathrm{~K}$ during TPD on the both samples with the resulting hydrogen atoms reacting with remaining methoxides to reform some methanol.

Differences are also observed in the photocatalytic activity of the platelets and bipyramids for the production of $\mathrm{HCOOCH}_{3}$ from methanol. As noted in the introduction, our previous results for the bipyramidal nanoparticles [13] indicated that the active sites for photocatalytic production of $\mathrm{HCOOCH}_{3}$ were located on the $\{101\}$ facets. The data in Figure 6 show that the initial rate of $\mathrm{HCOOCH}_{3}$ production on the platelets (regardless of size) during UV exposure is roughly $40 \%$ lower than that for the $10 \mathrm{~nm}$ bipyramids and $80 \%$ lower than that for the $25 \mathrm{~nm}$ bipyramids. Since the platelets expose a much lower fraction of $\{101\}$ facets relative to the bipyramids, these data provide additional evidence that the active sites for photochemical production of $\mathrm{HCOOCH}_{3}$ are on the exposed $\{101\}$ surfaces.

In addition to the relative exposure of $\{101\}$ and $\{001\}$ surfaces affecting the amount of $\mathrm{HCOOCH}_{3}$ produced, there are some more subtle differences in the photocatalytic activity of platelet and bipyramidal nanocrystals. Exposing the methanol-dosed bipyramidal nanocrystals to UV light also produced the following three trends in the TPD product distribution, each of which was less pronounced or nonexistent for the platelet nanocrystals: (1) photodecomposition of a portion of the $\mathrm{HCOOCH}_{3}$ product as evidenced by the amount of $\mathrm{HCOOCH}_{3}$ going through a 
maximum with UV exposure (see Figure 6), (2) an increase in $\mathrm{CH}_{4}$ production with $\mathrm{UV}$ exposure, especially for larger crystallites (see Figure 7), and (3) a decrease in the $\mathrm{CH}_{3} \mathrm{OCH}_{3}$ and $\mathrm{CH}_{2} \mathrm{O}$ yields with increasing UV exposure (compare Figures 3 and 5). The first two trends may be due to the fact that the rate of photochemical production of $\mathrm{HCOOCH}_{3}$ on the $\{101\}$ surfaces of the bipyramids is greater than that on the corresponding surfaces of the platelets (see Figure 6). This would cause the concentration of $\mathrm{HCOOCH}_{3}$ to saturate more quickly on the $\{101\}$ surfaces of the bipyramids at which point photodecomposition of the $\mathrm{HCOOCH}_{3}$, which produces adsorbed methyl groups, becomes the dominant reaction pathway. The faster rate of conversion of methanol to $\mathrm{HCOOCH}_{3}$ on the $\{101\}$ surfaces of the bipyramids compared to the platelets is likely related to the shape of the nanoparticles which causes photo-generated charge carriers in the bulk to have a higher probability of diffusing to a $\{101\}$ surface on the bipyramids. The third trend, however, is more difficult to explain. By similar reasoning, one might expect that the rate of photodecomposition of the methoxide groups on $\{001\}$ surfaces that react to produce $\mathrm{CH}_{3} \mathrm{OCH}_{3}$ or $\mathrm{CH}_{2} \mathrm{O}$ would be more rapid (at constant photon flux) on the platelets compared to the bipyramids, but just the opposite is observed.

We can only speculate as to the origin of this effect, but it is possible that the photocatalytic activity of the nanocrystals depends on cooperative effects between the $\{101\}$ and $\{001\}$ facets, in addition to individual contributions from each facet. A recent study by Liu et al. [29] provides some support for this possibility. In that study they measured the activity for photocatalytic water splitting for a series of truncated bipyramidal nanocrystals and found that the activity was highest on nanocrystals with $\sim 15 \%$ exposed $\{001\}$ facets, and decreased with higher or lower exposed fraction of these facets. Crystallites with platelet morphology similar to that used in the present study were found to be nearly inactive for the water splitting reaction. They argued that this trend 
was due to preferential migration of electrons and holes to separate facets on the $\mathrm{TiO}_{2}$ nanocrystals, as has been reported in several other papers [30-33]. Such separation of electrons and holes would reduce the rate of recombination resulting in an increase in quantum yield. This effect would be expected to be most pronounced when an electron-hole pair is generated in close proximity to a $\{101\}-\{001\}$ intersection. Just based on the nanocrystal geometries, on average electron-hole pairs generated near a $\{001\}$ facet on a bipyramid will be closer to one of these intersections than one near a $\{001\}$ facet on a platelet. Thus, facet driven carrier separation may, on a per area basis, enhance the photochemical activity of the bipyramid $\{001\}$ facets relative to those on the platelets. This may explain why photochemistry on the $\{001\}$ caps of the platelets used in this study appears to be proportionally lower than that of the bipyramids.

In the studies of facet-driven charge carrier separation mentioned above [29-33], the \{001\} and $\{101\}$ facets are reported as the primary destination of photo-generated holes and electrons, respectively. Since holes would be expected to participate in photo-oxidation reactions [34, 35], the $\{001\}$ facets would provide the sites for this reaction. This is in contrast to what was observed in the present study where photo-oxidation of methanol to $\mathrm{HCOOCH}_{3}$ was found to occur primarily on the $\{101\}$ facets. It should be noted, however, that the assignment of photogenerated carrier destinations in the literature have been based, for the most part, on solutionphase photodeposition of metal ions [31, 32] which may not be applicable to the vacuum conditions used in the present study. While suspended in solution the surfaces of the $\mathrm{A}-\mathrm{TiO}_{2}$ nanocrystals are almost certainly $-\mathrm{OH}$ terminated, which is not the case for the vacuum-annealed samples used here. Indeed XPS studies of the electronic band structure of $\mathrm{A}^{-} \mathrm{TiO}_{2}$ nanocrystals [36] correlated the abundance of $\{001\}$ facets with a lower conduction band minimum, which suggests that, at least in vacuum, photo-generated electrons may preferentially segregate to this 
surface. Alternatively, an indirect photo-oxidation mechanism, such as that described by D’Arienzo et al. [33], may enable oxidation on the electron-rich facets. While some questions may still remain about the ultimate destination of the electrons and holes, the results of this and previous studies still indicate that preferential segregation of electrons and holes to different exposed facets occurs, and that this affects the overall photocatalytic activity.

Finally, we consider the effect of nanocrystal size on photocatalytic activity. As can be seen in Figure $6 \mathrm{~b}$ and $7 \mathrm{~b}$, the $25 \mathrm{~nm}$ bipyramids are considerably more active than the $10 \mathrm{~nm}$ bipyramids for photochemical $\mathrm{HCOOCH}_{3}$ and $\mathrm{CH}_{4}$ production from methanol (or methoxide). Recall that these yields are normalized by the sample surface area. Since reactions occur on the surface but photon absorption occurs in the bulk, the normalized yield might be expected to scale with the volume-to-surface area ratio of the nanoparticles (at least until the nanocrystal size approaches the photon penetration depth). The size dependence of photocatalytic activity on the bipyramids follows this pattern closely enough to suggest that the volume-to-surface area ratio plays a role.

In contrast to the bipyramids, the platelets show no discernable size dependence for photochemical reactions. As discussed above, charge carriers generated further away from the photochemically active $\{101\}$ surfaces (e.g., near the center of a platelet) are more likely to recombine prior to diffusing to this surface (precluding surface reactions) than those generated near this surface. For comparison, no point in the $25 \mathrm{~nm}$ bipyramid nanocrystal is further than $\sim 6$ $\mathrm{nm}$ from a $\{101\}$ facet, while the center of even the smallest platelets is $\sim 7 \mathrm{~nm}$ from one of these facets. It may even be that the volume near the center of the larger platelets is ineffective in providing photo-generated carriers to the $\{101\}$ facets. In this case, the extra volume for photon absorption gained by the $18 \mathrm{~nm}$ and $22 \mathrm{~nm}$ platelets would be partially offset by the expanding 
'dead volume' near the center. This would explain the lack of size effects for $\mathrm{HCOOCH}_{3}$ production on the platelets. Alternatively, perhaps any size effects are too small to be observed over the $\sim 3$-fold volume increase from $14 \mathrm{~nm}$ to $22 \mathrm{~nm}$ platelets, while the $\sim 15$-fold volume increase from the $10 \mathrm{~nm}$ to $25 \mathrm{~nm}$ bipyramids makes these effects noticeable.

\section{Conclusions}

The results of this study of the reactivity of methanol on $\mathrm{A}-\mathrm{TiO}_{2}$ nanoparticles with platelet and bipyramidal morphology further demonstrates the crystal-plane dependent reactivity of A$\mathrm{TiO}_{2}$. In particular, we further confirmed our previous finding that the active sites for the production of $\mathrm{CH}_{3} \mathrm{OCH}_{3}$ from adsorbed methoxide groups are located on the $\{001\}$ surfaces while those for the photochemical oxidation and coupling of adsorbed methanol and methoxide groups to produce $\mathrm{HCOOCH}_{3}$ are located on the $\{101\}$ surfaces. This causes the platelet nanocrystals to be more active for the production of $\mathrm{CH}_{3} \mathrm{OCH}_{3}$, and the bipyramidal nanocrystals to be more active for the photochemical reaction that produces $\mathrm{HCOOCH}_{3}$. A dependence of the reactivity on the facet size was also observed with the smallest $\{001\}$ facets being active for the coupling of methoxide groups to produce $\mathrm{CH}_{3} \mathrm{OCH}_{3}$ which is not observed on the extended (001) surfaces of macroscopic $\mathrm{A}-\mathrm{TiO}_{2}$ single crystals. This observation indicates that the complex reconstructions which occur on the $\mathrm{A}-\mathrm{TiO}_{2}(001)$ surface may be facet-size dependent.

The bipyramidal nanocrystals were also found to be more active than the platelet nanocrystals for all photochemical reactions observed, including those occurring on the $\{001\}$ planes, such as the depletion of methoxide precursors which would otherwise react to form $\mathrm{CH}_{3} \mathrm{OCH}_{3}$ on this surface. This points to a difference in the intrinsic photochemical activity of both morphologies, rather than a simple matter of active site abundance and is consistent with the 
idea that the ratio of exposed $\{101\}$ and $\{001\}$ surface area plays a key role in $\mathrm{TiO}_{2}$ photocatalysis as suggested in several previous studies [29-33]. Specifically, facet-driven charge carrier separation may play a role in enhancing the photocatalytic activity of bipyramidal nanocrystals relative to platelet nanocrystals. Thus, the relative proximity of dissimilar planes

may be an important parameter for the design of highly-active photocatalytic anatase nanoparticles. It was also shown that larger bipyramids have increased photocatalytic activity over smaller bipyramids, indicating that the ratio of volume to surface area is important. Thus, there may be an optimal particle size for any given photochemical reaction which balances higher photon absorption for larger sizes with the higher surface area and more active sites at lower sizes.

Finally this study highlights the utility of thin films of well-defined nanocrystallites as model catalysts in fundamental studies of structure-activity relationships for metal oxides and further demonstrates how this approach can help bridge the materials gap between studies of single crystals and high surface area catalytic materials.

\section{Acknowledgements}

Funding for this study was provided by the U.S. Department of Energy, Office of Science, Office of Basic Energy Sciences under grant no. DE-FG02-04ER15605.

\section{References}

[1] J.M. Vohs, Chem. Rev., 113 (2013) 4136-4163.

[2] M.A. Barteau, J. Vac. Sci. Technol., A, 11 (1993) 2162-2168.

[3] M.A. Barteau, Chem. Rev., 96 (1996) 1413-1430. 
[4] H. Idriss, M.A. Barteau, Adv. Catal., 45 (2000) 261-331.

[5] A.K.P. Mann, Z.L. Wu, F.C. Calaza, S.H. Overbury, ACS Catal., 4 (2014) 2437-2448.

[6] S. Agarwal, L. Lefferts, B.L. Mojet, Chemcatchem, 5 (2013) 479-489.

[7] G. Centi, R. Passalacqua, S. Perathoner, D.S. Su, G. Weinberg, R. Schlogl, PCCP, 9 (2007) 4930-4938.

[8] G. Centi, S. Perathoner, Coord. Chem. Rev., 255 (2011) 1480-1498.

[9] T. Ressler, B.L. Kniep, I. Kasatkin, R. Schlogl, Angew. Chem. Int. Ed., 44 (2005) 47044707.

[10] H.X. Mai, L.D. Sun, Y.W. Zhang, R. Si, W. Feng, H.P. Zhang, H.C. Liu, C.H. Yan, J. Phys. Chem. B, 109 (2005) 24380-24385.

[11] S.R. Zhang, L. Nguyen, Y. Zhu, S.H. Zhan, C.K. Tsung, F. Tao, Acc. Chem. Res., 46 (2013) 1731-1739.

[12] H.J. Freund, N. Ernst, T. Risse, H. Hamann, G. Rupprechter, Phys. Status Solidi A, 187 (2001) 257-274.

[13] D.A. Bennett, M. Cargnello, T.R. Gordon, C.B. Murray, J.M. Vohs, PCCP, 17 (2015) 17190-17201.

[14] T.R. Gordon, M. Cargnello, T. Paik, F. Mangolini, R.T. Weber, P. Fornasiero, C.B. Murray, J. Am. Chem. Soc., 134 (2012) 6751-6761.

[15] K.S. Kim, M.A. Barteau, J. Catal., 125 (1990) 353-375.

[16] K.S. Kim, M.A. Barteau, W.E. Farneth, Langmuir, 4 (1988) 533-543.

[17] M.A. Henderson, S. Otero-Tapia, M.E. Castro, Faraday Discuss., 114 (1999) 313-329.

[18] K.S. Kim, M.A. Barteau, Surf. Sci., 223 (1989) 13-32. 
[19] C.B. Xu, W.S. Yang, Q. Guo, D.X. Dai, M.D. Chen, X.M. Yang, J. Am. Chem. Soc., 136 (2014) 602-605.

[20] R. Hengerer, B. Bolliger, M. Erbudak, M. Gratzel, Surf. Sci., 460 (2000) 162-169.

[21] U. Diebold, Surf. Sci. Rep., 48 (2003) 53-229.

[22] G.S. Herman, M.R. Sievers, Y. Gao, Phys. Rev. Lett., 84 (2000) 3354-3357.

[23] R.E. Tanner, Y. Liang, E.I. Altman, Surf. Sci., 506 (2002) 251-271.

[24] Y. Liang, S.P. Gan, S.A. Chambers, E.I. Altman, Phys. Rev. B, 63 (2001).

[25] U. Diebold, N. Ruzycki, G.S. Herman, A. Selloni, Catal. Today, 85 (2003) 93-100.

[26] M. Lazzeri, A. Selloni, Phys. Rev. Lett., 87 (2001).

[27] V.S. Lusvardi, M.A. Barteau, W.E. Farneth, J. Catal., 153 (1995) 41-53.

[28] M.V. Ganduglia-Pirovano, A. Hofmann, J. Sauer, Surf. Sci. Rep., 62 (2007) 219-270.

[29] C. Liu, X.G. Han, S.F. Xie, Q. Kuang, X. Wang, M.S. Jin, Z.X. Xie, L.S. Zheng, ChemAsian J, 8 (2013) 282-289.

[30] W. Chen, Q. Kuang, Q.X. Wang, Z.X. Xie, Rsc Adv, 5 (2015) 20396-20409.

[31] N. Murakami, Y. Kurihara, T. Tsubota, T. Ohno, J. Phys. Chem. C, 113 (2009) 3062-3069.

[32] T. Ohno, K. Sarukawa, M. Matsumura, New J. Chem., 26 (2002) 1167-1170.

[33] M. D'Arienzo, J. Carbajo, A. Bahamonde, M. Crippa, S. Polizzi, R. Scotti, L. Wahba, F. Morazzoni, J. Am. Chem. Soc., 133 (2011) 17652-17661.

[34] C.M. Friend, Chem. Rec., 14 (2014) 944-951.

[35] M.A. Henderson, Surf. Sci. Rep., 66 (2011) 185-297.

[36] J. Pan, G. Liu, G.M. Lu, H.M. Cheng, Angew. Chem. Int. Ed., 50 (2011) 2133-2137. 


\section{Figure Captions}

Figure 1. Schematic showing the shape and exposed crystal planes of the (a) platelet and (b) bipyramidal anatase nanocrystals. Both forms present exclusively $\{101\}$ and $\{001\}$ surfaces, but the $\{001\}$ surfaces account for a larger fraction of the platelet nanocrystals.

Figure 2. TEM micrographs of the as-synthesized (a) platelet and (b) bipyramidal anatase nanocrystals, and SEM micrographs of the (c) platelet and (d) bipyramidal nanocrystal thin films dispersed onto oxidized silicon substrates which were used in reactivity studies.

Figure 3. TPD results obtained from (a) $14 \mathrm{~nm}$ platelet and (b) $10 \mathrm{~nm}$ bipyramidal nanocrystal thin films dosed with $2.5 \mathrm{~L}$ of methanol at $160 \mathrm{~K}$. Desorption intensities have been scaled to account for mass spectrometer sensitivity and difference in surface area.

Figure 4. Dimethyl ether yield following a $2.5 \mathrm{~L}$ methanol dose as a function of nanocrystal size and shape. All yields have been normalized to account for differences in surface area.

Figure 5. TPD results obtained from (a) $14 \mathrm{~nm}$ platelet and (b) $10 \mathrm{~nm}$ bipyramidal nanocrystal thin films dosed with $2.5 \mathrm{~L}$ of methanol at $160 \mathrm{~K}$ and exposed to $3 \mathrm{~h}$ of $\mathrm{UV}$ 
illumination. Desorption intensities have been scaled to account for mass spectrometer sensitivity and difference in surface area.

Figure 6. Methyl formate yield following a 2.5 L methanol dose from (a) platelet and (b) bipyramidal nanocrystal thin films as a function of UV exposure time and nanocrystal size and shape. All yields have been normalized to account for differences in surface area.

Figure 7. Methane yield following a 2.5 L methanol dose from the (a) platelet and (b) bipyramidal nanocrystal thin films as a function of UV exposure time and nanocrystal size and shape. All yields have been normalized to account for differences in surface area.

Figure 8. Dimethyl ether yield normalized by the available $\{001\}$ surface area as a function of the area of a single (001) facet. 
Figures

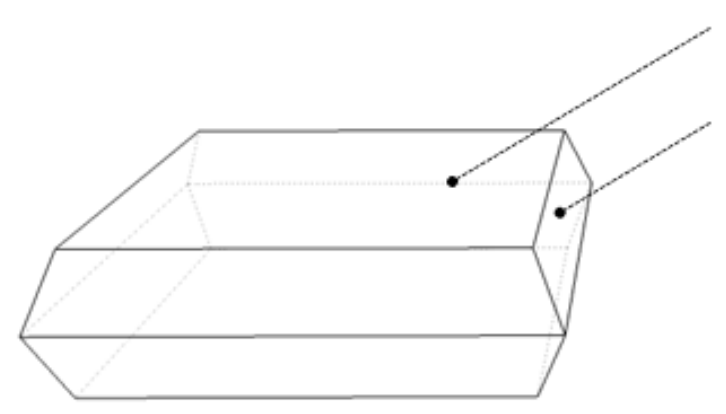

a. Platelet

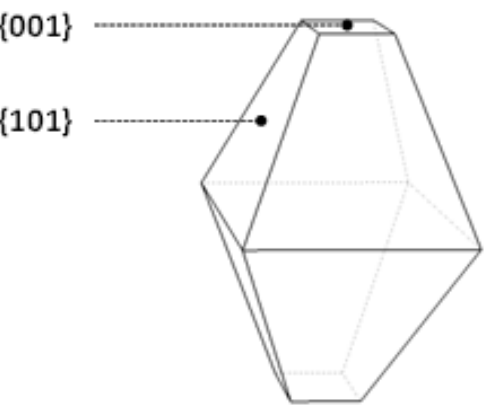

b. Bipyramid

Figure 1. Schematic showing the shape and exposed crystal planes of the (a) platelet and (b) bipyramidal anatase nanocrystals. Both forms present exclusively $\{101\}$ and $\{001\}$ surfaces, but the $\{001\}$ surfaces account for a larger fraction of the platelet nanocrystals. 


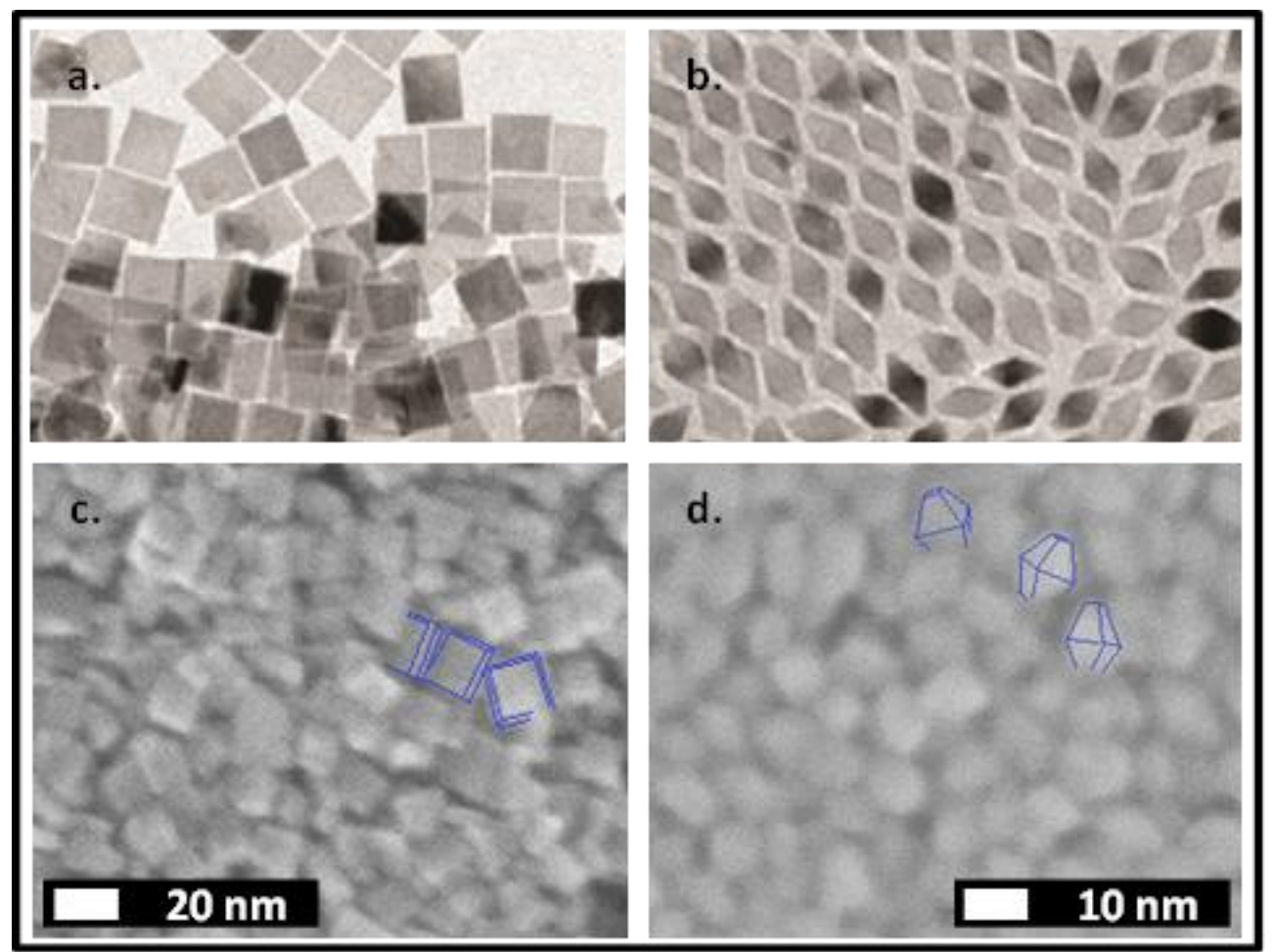

Figure 2. TEM micrographs of the as-synthesized (a) platelet and (b) bipyramidal anatase nanocrystals, and SEM micrographs of the (c) platelet and (d) bipyramidal nanocrystal thin films dispersed onto oxidized silicon substrates which were used in reactivity studies. 

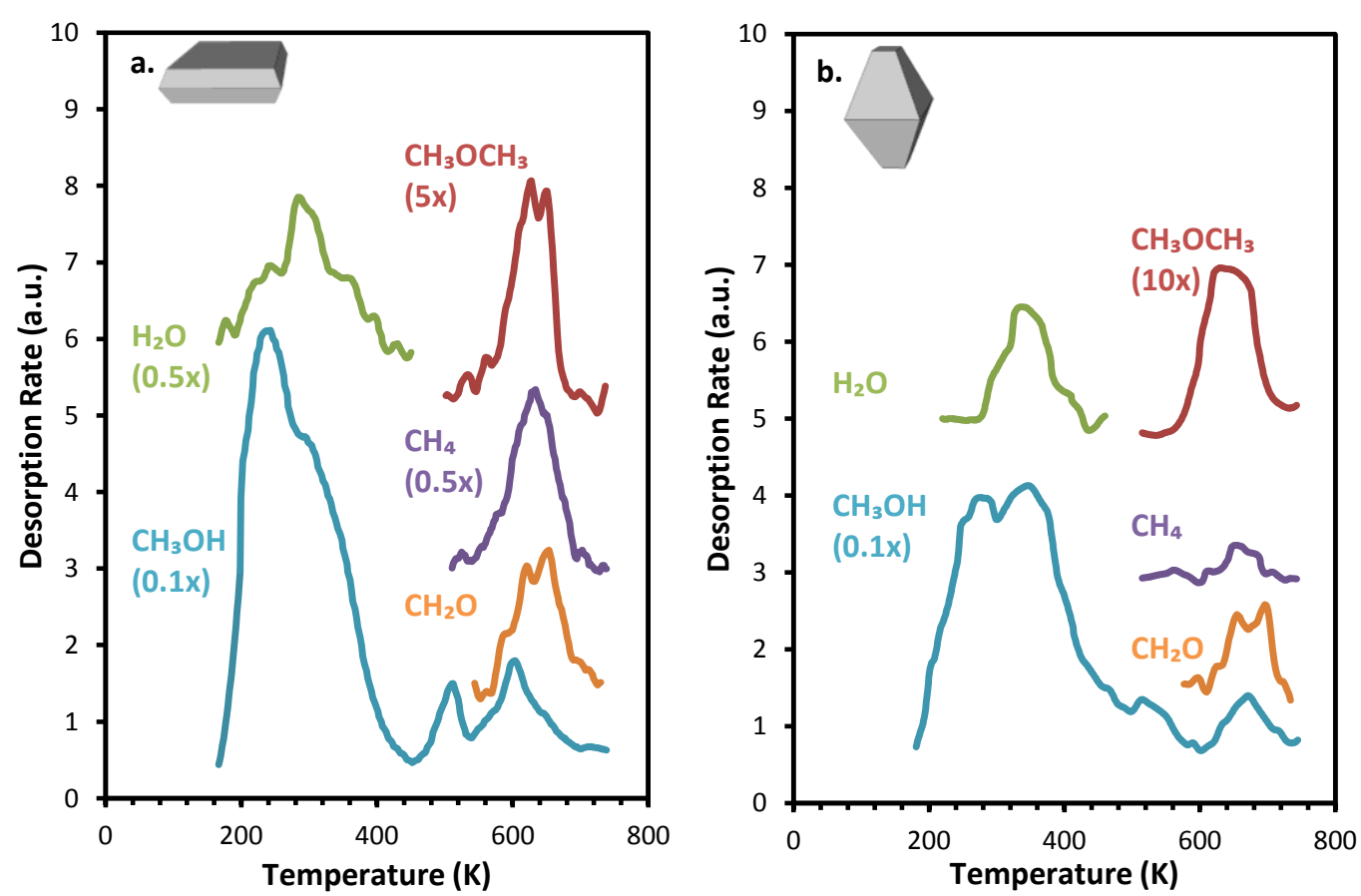

Figure 3. TPD results obtained from (a) $14 \mathrm{~nm}$ platelet and (b) $10 \mathrm{~nm}$ bipyramidal nanocrystal thin films dosed with $2.5 \mathrm{~L}$ of methanol at $160 \mathrm{~K}$. Desorption intensities have been scaled to account for mass spectrometer sensitivity and difference in surface area. 


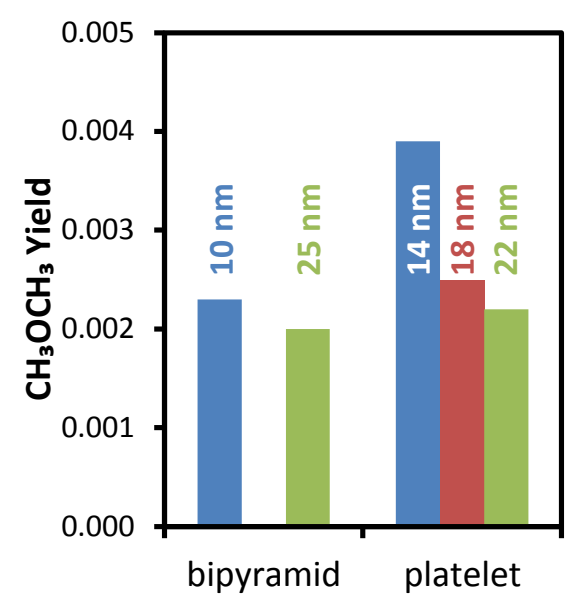

Figure 4. Dimethyl ether yield following a $2.5 \mathrm{~L}$ methanol dose as a function of nanocrystal size and shape. All yields have been normalized to account for differences in surface area. 

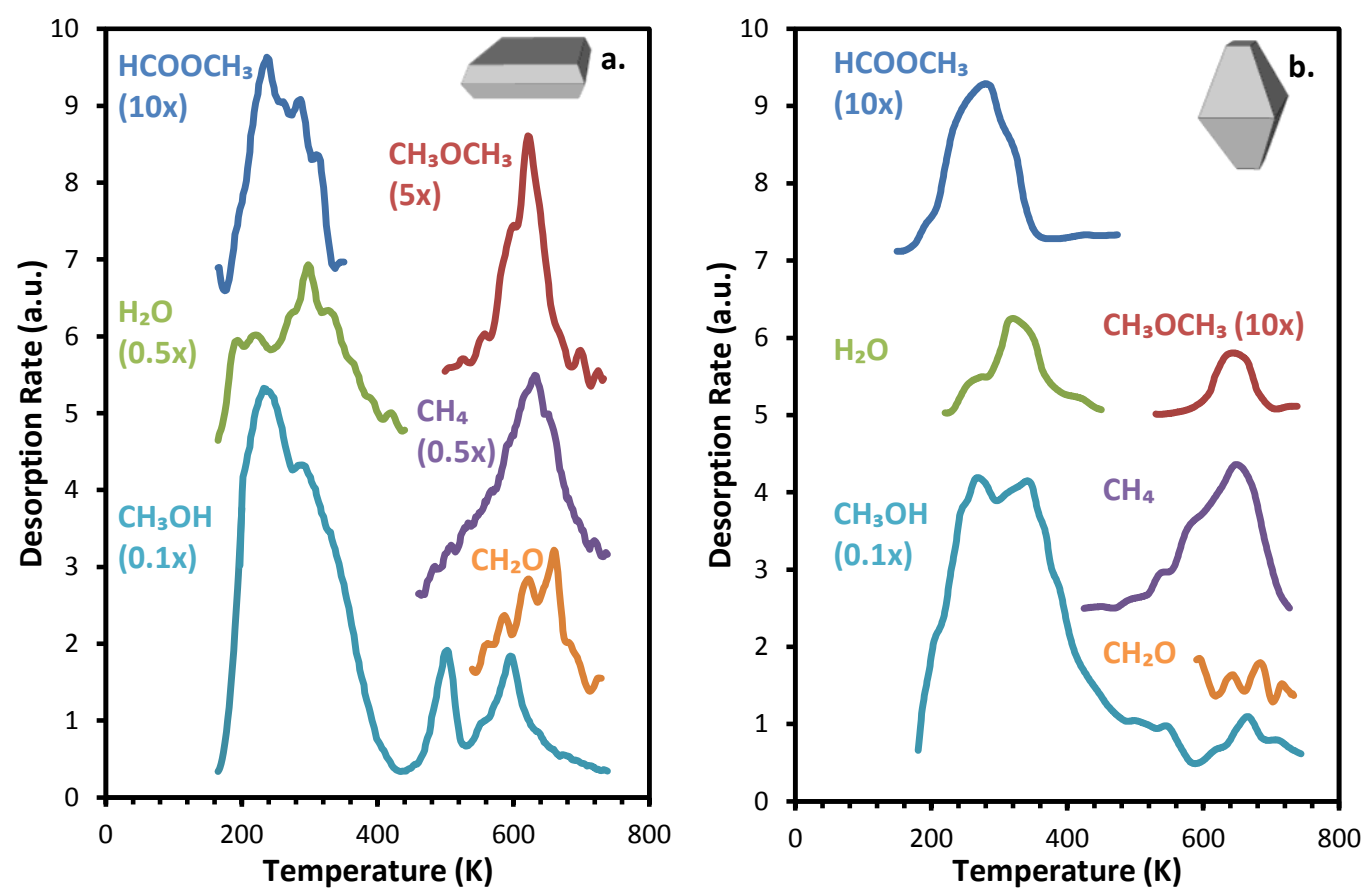

Figure 5. TPD results obtained from (a) $14 \mathrm{~nm}$ platelet and (b) $10 \mathrm{~nm}$ bipyramidal nanocrystal thin films dosed with $2.5 \mathrm{~L}$ of methanol at $160 \mathrm{~K}$ and exposed to $3 \mathrm{~h}$ of UV illumination. Desorption intensities have been scaled to account for mass spectrometer sensitivity and difference in surface area. 

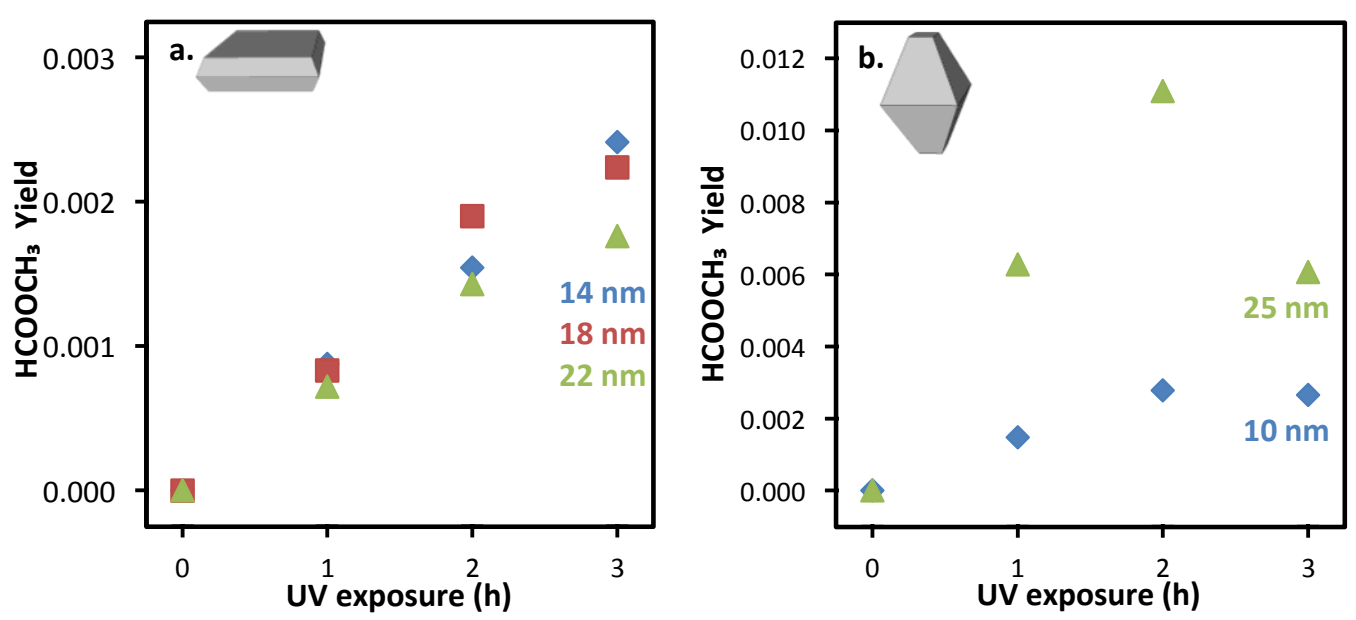

Figure 6. Methyl formate yield following a 2.5 L methanol dose from (a) platelet and (b) bipyramidal nanocrystal thin films as a function of UV exposure time and nanocrystal size and shape. All yields have been normalized to account for differences in surface area. 

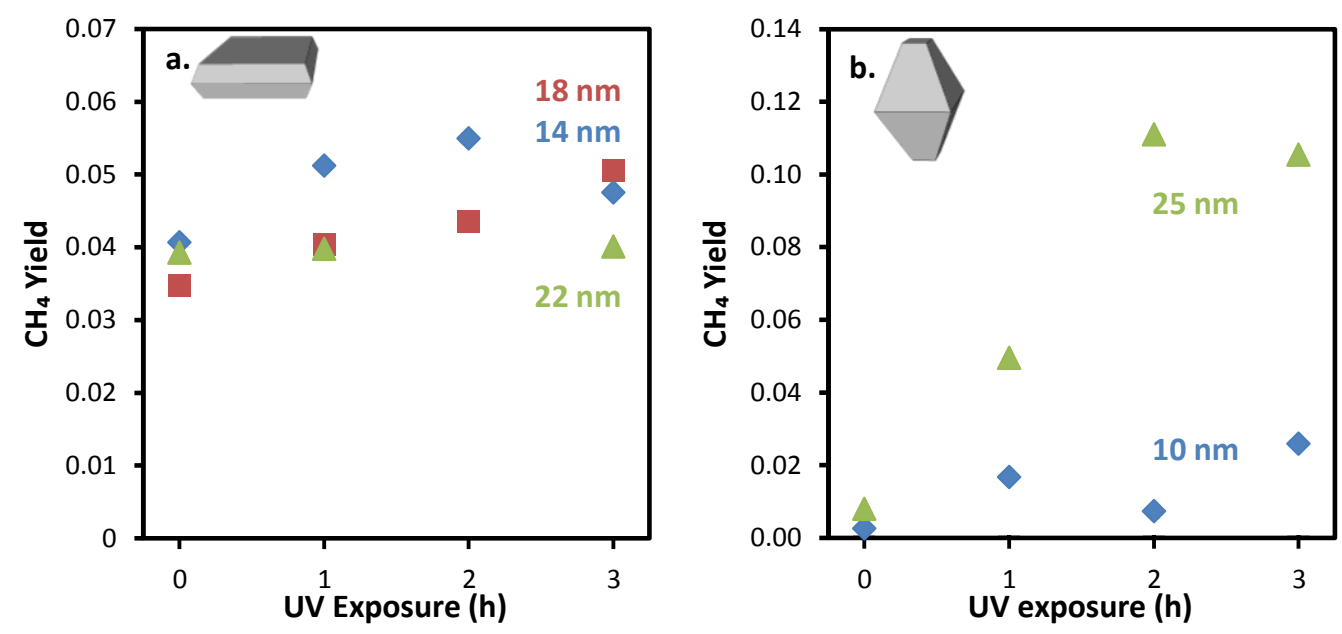

Figure 7. Methane yield following a 2.5 L methanol dose from the (a) platelet and (b) bipyramidal nanocrystal thin films as a function of UV exposure time and nanocrystal size and shape. All yields have been normalized to account for differences in surface area. 


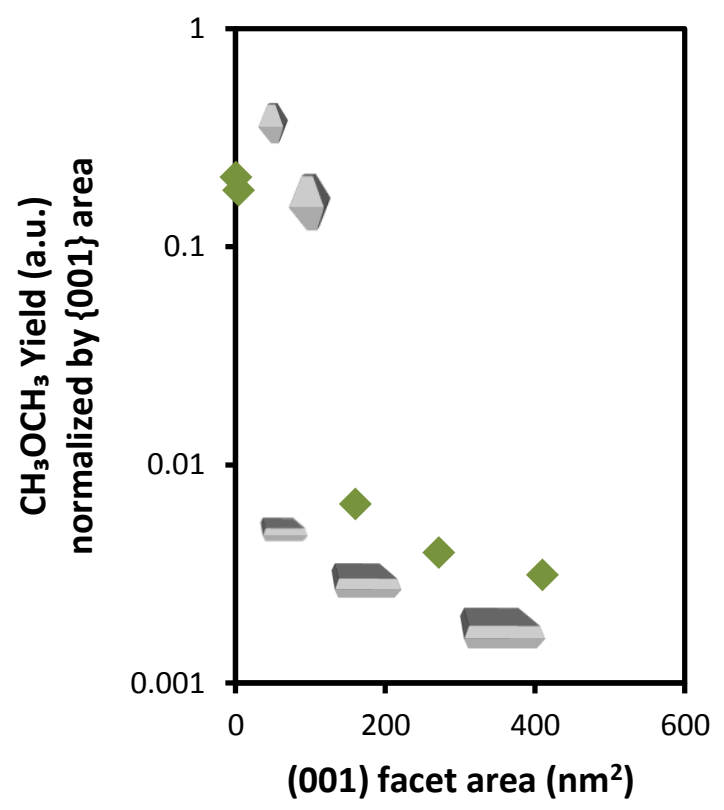

Figure 8. Dimethyl ether yield normalized by the available $\{001\}$ surface area as a function of the area of a single (001) facet. 
Table

Table 1. Product yields for $2.5 \mathrm{~L}$ methanol dose

\begin{tabular}{|c|r|r|r|r|r|}
\hline $\begin{array}{c}\text { Nanocrystal Size and } \\
\text { Shape }\end{array}$ & $\mathrm{CH}_{3} \mathrm{OCH}_{3}$ & \multicolumn{1}{c|}{$\mathrm{CH}_{\mathbf{2}} \mathbf{O}$} & \multicolumn{1}{c|}{$\mathbf{C H}_{4}$} & \multicolumn{1}{|c|}{$\begin{array}{c}\text { High-temp } \\
\mathrm{CH}_{3} \mathbf{O H}\end{array}$} & $\mathbf{H}_{2} \mathbf{O}$ \\
\hline \hline $14 \mathrm{~nm}$ platelets & 0.0039 & 0.018 & 0.040 & 0.025 & 0.053 \\
\hline $18 \mathrm{~nm}$ platelets & 0.0025 & 0.021 & 0.034 & 0.033 & 0.023 \\
\hline $22 \mathrm{~nm}$ platelets & 0.0022 & 0.019 & 0.039 & 0.027 & 0.025 \\
\hline $10 \mathrm{~nm}$ bipyramids & 0.0023 & 0.012 & 0.003 & 0.031 & 0.011 \\
\hline $25 \mathrm{~nm}$ bipyramids & 0.0020 & 0.029 & 0.008 & 0.043 & 0.032 \\
\hline
\end{tabular}

